\title{
Measurement Uncertainty of Nano Bionix Universal Testing System
}

\author{
Chung-Lin $\mathrm{Wu}^{1,2}$, Fu-Lung Pan², Chin-Fen Tuan², \\ Sheng-Jui Chen ${ }^{2}$, Weileun Fang ${ }^{1}$ and Ming-Chuen Yip ${ }^{1, *}$ \\ ${ }^{1}$ Department of Power Mechanical Engineering, National Tsing Hua University, \\ 101 Kuang Fu Road, Sec. 2, Hsinchu, Taiwan 30013 \\ ${ }^{2}$ Center for Measurement Standards, Industrial Technology Research Institute, \\ 321 Kuang Fu Road, Sec. 2, Hsinchu, Taiwan 30013
}

(Received August 15, 2008; accepted October 8, 2008)

Key words: uncertainty, force, displacement measurement

The main purpose of this research is to determine the uncertainties of force and displacement measurements for MTS Nano Bionix Universal Testing System (UTM). This article can be used as basis for calculating measurement uncertainty in performing material tests. Standard weights are used to calibrate the force of the testing system. In addition, an optical method is adopted to evaluate the displacement uncertainty of the system. We adopted the method suggested in ISO to calculate the uncertainty of this system. The relative expanded uncertainty of force measurement within the range of 10 to $200 \mathrm{mN}$ is $2.07 \times 10^{-3}$ with a $95 \%$ confidence level. The expanded uncertainty of displacement measurement within the range of 0 to $88 \mathrm{~mm}$ is $1.4 \times 10^{-5} \mathrm{~m}$ with a $95 \%$ confidence level.

\section{Introduction}

The Nano Bionix Universal Testing System (UTM) is used to measure the mechanical properties of materials. It can be used to measure tension, compression, adhesion, threeor four-point bending, and dynamic mechanical tests. It consists of a frame and moving crosshead, but in place of the load cell is a Nano Mechanical Actuating Transducer (NMAT). The specifications of this system are as follows. ${ }^{(1)}$ This testing system is performed with a load range of $0-500 \mathrm{mN}$ and a crosshead extension range of $0-150 \mathrm{~mm}$. Its dynamic frequency range (CDA option) is from $0.1 \mathrm{~Hz}$ to $2.5 \mathrm{kHz}$.

For realizing the capability of the Nano Bionix UTM, it is important to calculate the ability of this system. The purpose of this article is to estimate the uncertainty of the Nano Bionix Universal Testing System (MTS Systems Corp., Oak Ridge, TN, USA). The method suggested in ISO $\mathrm{GUM}^{(2)}$ was adopted to calculate the system uncertainty. The relative expanded uncertainties of force and displacement measurements are

*Corresponding author: e-mail: mcyip@pme.nthu.edu.tw 
evaluated and calculated in this article. The standard weights used to calibrate the force of Nano UTM can be traced back to International Prototype Kilogram (IPK). An optical interferometer system was established to calibrate the displacement measurement. In addition, the elastic modulus of the material was derived from the stress-strain theory.

To calculate the elastic modulus for Nano UTM, polydimethylsiloxane (PDMS) was adopted in this paper. It is a nontoxic and silicon-based organic polymer that has been widely used in biotechnology and microdevices. ${ }^{(3-5)}$ The pure PDMS of Dow Corning Sylgard 184 consisted of a base and a curing agent. The curing temperature was set at $100^{\circ} \mathrm{C}$ for one hour. The specimens were cut into pieces that were $2 \mathrm{~mm}$ wide and the gauge length was $20 \mathrm{~mm}$. The mechanical properties of PDMS materials were measured by Nano UTM. The quasi-static tensile strength tests were conducted at a strain rate of 0.001/s for all the specimens. The elastic modulus was determined from the measured stress-strain curve.

\section{Calibration Setup}

To calibrate the force and displacement measurement of Nano UTM, the standard weights traced back to IPK and the setup of an optical interferometer were used for the experiments in this research.

\subsection{Force calibration}

The Nano UTM possesses an independent loading system. The standard weights put on the platform of NMAT were used to calibrate the loading system as shown in Fig. 1. The standard weights and the readouts of the loading system were utilized for intercomparison. By using the static force balance, the formula is expressed as

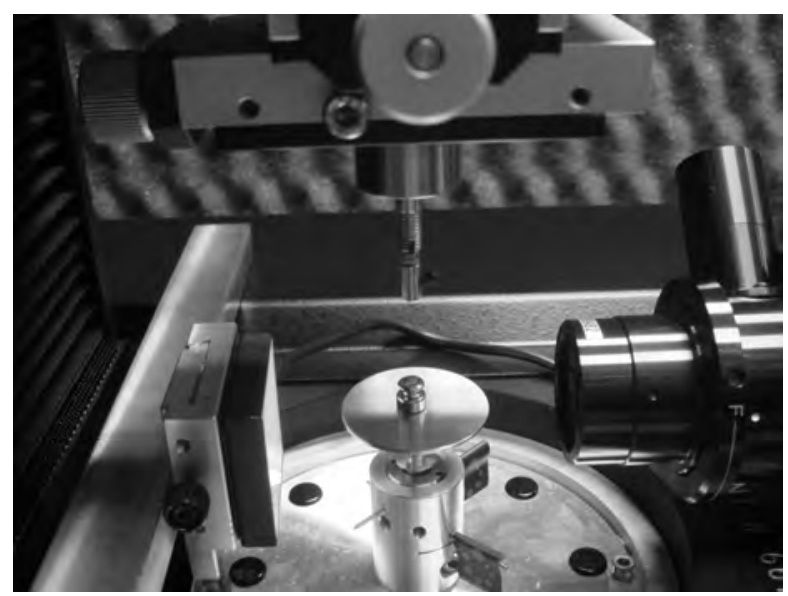

Fig. 1. One gram standard weight. 


$$
F=m g-B,
$$

where $F$ is the balance force, $m$ is the mass of standard weight, $g$ is the gravity acceleration, and $B$ is the air buoyancy.

From this formula, the gravity and air buoyancy should be considered with different temperatures and pressures. Because of the zero linear drift of the instrument, the ABA weighing cycles of calibration design were adopted in this system. The standard weights of E2 class in the range of 1-20 g correspond to the rule of International Organization of Legal Metrology (OIML) R111. This weight was set as the working standard and can be traced to SI unit as shown in Fig. 2.

\subsection{Displacement calibration}

The displacement sensing part of UTM is calibrated using a commercial optical heterodyne interferometer. The light source for the interferometer is a 633-nm twofrequency beam consisting of two orthogonally polarized frequency components. The resolution of the heterodyne interferometer is $10 \mathrm{~nm}$. Figure 3 is the scheme of the calibration setup. The design of the optical path is shown as Fig. 4. The calibration procedure is to move the crosshead of UTM step by step and record the resulting displacement measured both by the displacement sensor within the UTM and the external interferometer. The uncertainty of UTM displacement sensor is then evaluated from the 2 sets of displacement data.

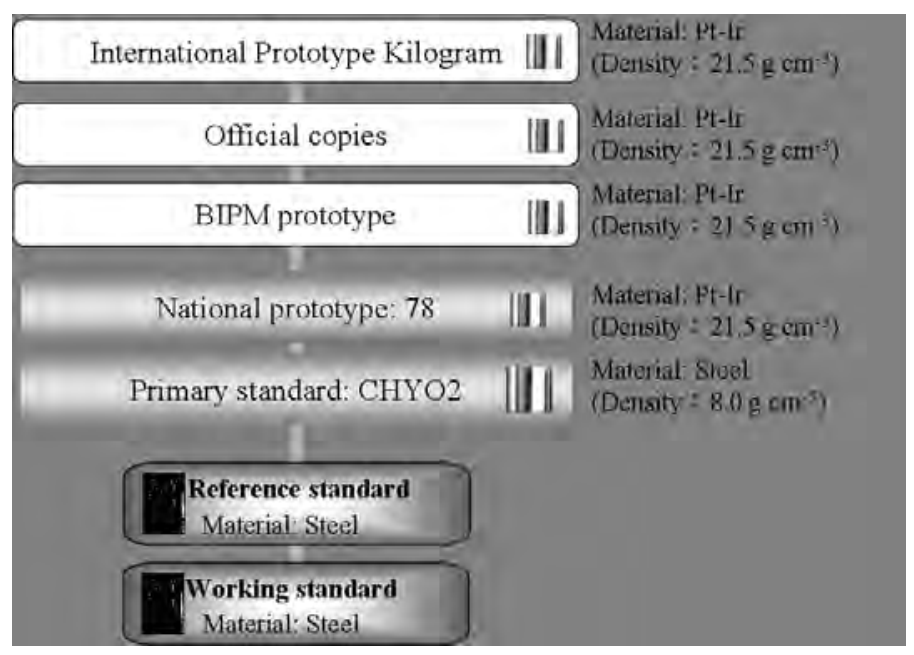

Fig. 2. Traceability chart. 


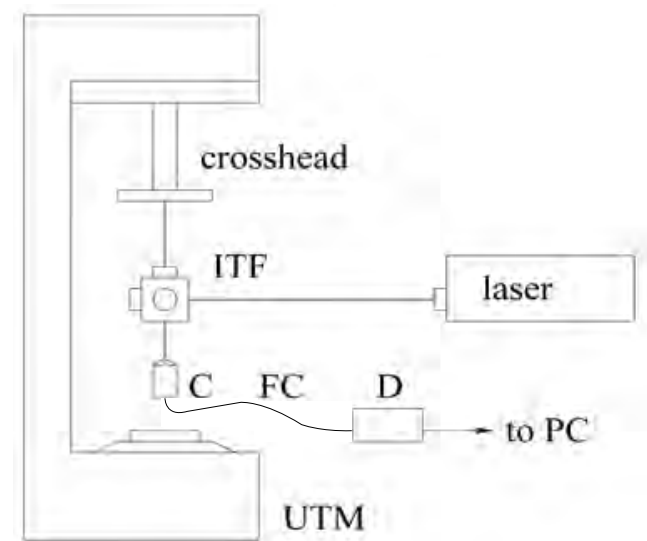

ITF: Heterodyne interferometer

C: Collimator

FC: Fiber cable

D: Photoreceiver

Fig. 3. Scheme of calibration setup.

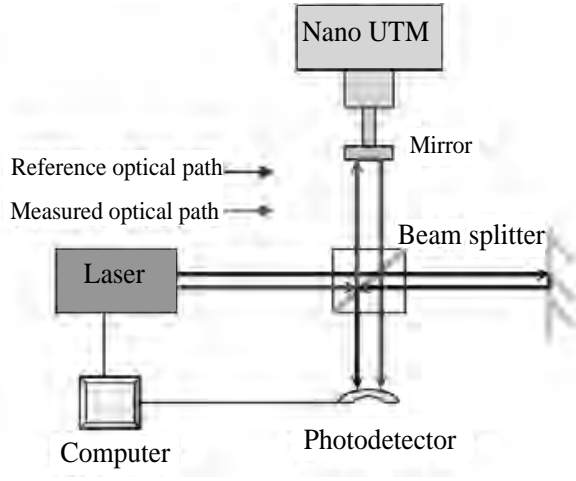

Reference mirror

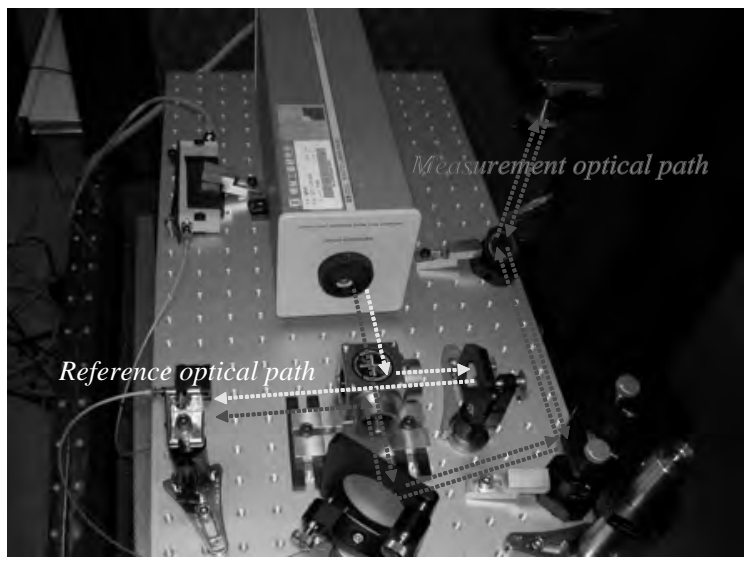

Fig. 4. Design of optical path.

\section{Uncertainty Evaluation and Measurement}

To evaluate the uncertainty of force and displacement measurement, the factors such as the repeatability uncertainty of the measurement system, the indication error of the system, and environmental effects should be considered. 


\subsection{Uncertainty evaluation of force}

A series of traceable standard weights was used to calibrate the loading of Nano UTM in the range of 10-200 mN. Since the effect of gravity was considered, the loading can be transformed into the force. The uncertainty of force was caused by three different factors. The first one is uncertainty of weights and the second is repeatability of the measurement system. The indication error of the measurement system is also a factor that will eventually be affected.

\subsubsection{Standard uncertainty that resulted from uncertainty of standard weights $\left(u_{1}\right)$}

The standard weights correspond to the rule of OIML R111. The density of weights is $8000 \mathrm{~kg} / \mathrm{m}^{3}$ and the density of air is $1.2 \mathrm{~kg} / \mathrm{m}^{3}$. The gravity acceleration of the lab is approximately $9.8 \mathrm{~m} / \mathrm{s}^{2}$. The data are shown in Table 1 .

\subsubsection{Standard uncertainty that resulted from the repeatability uncertainty of measurement system $\left(u_{2}\right)$}

The standard uncertainty that resulted from the repeatable measurement system can be represented using the calibrated data standard.

\subsubsection{Standard uncertainty that resulted from indication error of the loading system $\left(u_{3}\right)$}

For convenient calculation, it is not considered to modify the indication error of the loading system. However, it is easy to calculate the uncertainty of measurement including the indication error. The rectangular distribution was adopted when calculating the probability distribution of indication error as shown in Table 1.

Table 1

Uncertainty evalutation of Nano UTM.

\begin{tabular}{|c|c|c|c|c|c|c|c|c|c|}
\hline \multirow[b]{2}{*}{$\begin{array}{l}\text { Force } \\
(\mathrm{mN})\end{array}$} & \multicolumn{4}{|c|}{ Standard weights } & \multicolumn{2}{|c|}{$\begin{array}{l}\text { Loading system } \\
\text { (Nano UTM) }\end{array}$} & \multirow[b]{2}{*}{$\begin{array}{c}\text { Deviation } \\
\text { error } \\
(\mathrm{mN})\end{array}$} & \multirow[b]{2}{*}{\begin{tabular}{|c|} 
Standard \\
uncertainty of \\
deviation \\
error \\
$(\mathrm{mN})$ \\
$u_{3}$
\end{tabular}} & \multirow[b]{2}{*}{$\begin{array}{c}\text { Combined } \\
\text { standard } \\
\text { uncertainty } \\
(\mathrm{mN}) \\
u_{\mathrm{F} 1}\end{array}$} \\
\hline & Mass (g) & $\begin{array}{l}\text { Standard } \\
\text { uncertainty } \\
\text { (g) }\end{array}$ & $\begin{array}{c}\text { Force from } \\
\text { standard } \\
\text { weights } \\
(\mathrm{mN})\end{array}$ & \begin{tabular}{|c} 
Standard \\
uncertainty \\
that resulted \\
from \\
uncertainty \\
of standard \\
weights \\
$(\mathrm{mN})$ \\
$u_{1}$
\end{tabular} & $\begin{array}{l}\text { Average of } \\
\text { system } \\
\text { calibration } \\
(\mathrm{mN})\end{array}$ & $\begin{array}{c}\text { Standard } \\
\text { deviation of } \\
\text { calibration } \\
(\mathrm{mN}) \\
u_{2}\end{array}$ & & & \\
\hline 200 & 20.000003 & 0.005584 & \begin{tabular}{|l|}
195.75345 \\
\end{tabular} & 0.05465 & 196.44247 & 0.01446 & 0.68901 & 0.19890 & 0.20678 \\
\hline 100 & 10.0000074 & 0.002759 & 97.87678 & 0.02700 & 98.20598 & 0.00803 & 0.32920 & 0.09503 & 0.09912 \\
\hline 50 & 5.0000130 & 0.003081 & 48.93848 & 0.03016 & 49.05513 & 0.00220 & 0.11664 & 0.03367 & 0.04526 \\
\hline 20 & 2.0000021 & 0.001633 & 19.57536 & 0.01598 & 19.61834 & 0.02299 & 0.04298 & 0.01241 & 0.03063 \\
\hline 10 & 1.0000044 & 0.001574 & 9.78771 & 0.01541 & 9.80096 & 0.03160 & 0.03124 & 0.00382 & 0.03536 \\
\hline
\end{tabular}


Finally, the combined standard uncertainty of the measurement system can be expressed as

$$
u_{F 1}=\sqrt{u_{1}^{2}+u_{2}^{2}+u_{3}^{2}} .
$$

The results of the combined standard uncertainty in the range of $10-200 \mathrm{mN}$ are shown in Table 1. The expanded uncertainty of force with a $95 \%$ confidence level is equal to $0.207 \mathrm{mN}$.

\subsection{Uncertainty evaluation of displacement measurement}

The setup of displacement measurement is described above. The uncertainty of the measurement displacement is affected by six different factors as follows.

\subsubsection{Uncertainty that resulted from zero drift of the laser interferometer in a} static state $\left(u_{4}\right)$

Because of environmental effects such as air flow, humidity and excited oscillation for instruments, the interferometer drift would be induced. The system was controlled at a frequency of $500 \mathrm{~Hz}$. It recorded the averages of forty zero points in five seconds. Based on Table 2, the standard uncertainty of zero drift in a static state can be calculated from the standard deviation $S\left(X_{n}\right)$.

$$
S\left(X_{n}\right)=\sqrt{\frac{\sum_{n=1}^{40}\left(X_{n}-\overline{X_{n}}\right)^{2}}{40-1}}=4.37 \times 10^{-8}(\mathrm{~m})
$$

Here, $X_{n}$ : individual recorder value of zero drift $(n=1-40), \bar{X}_{n}$ : average value of $X_{n}$.

It was assumed that uncertainty from zero drift of the laser interferometer equals the standard deviation $\left(u_{4}=S\left(X_{n}\right)\right)$, and the degree of freedom is $39\left(v_{4}\right)$.

Table 2

Individual recorder value of zero drift.

\begin{tabular}{rrrr}
\hline $9.319 \mathrm{E}-08$ & $1.961 \mathrm{E}-08$ & $-1.160 \mathrm{E}-08$ & $1.871 \mathrm{E}-08$ \\
$3.323 \mathrm{E}-08$ & $8.551 \mathrm{E}-09$ & $-8.288 \mathrm{E}-08$ & $6.047 \mathrm{E}-09$ \\
$2.818 \mathrm{E}-08$ & $5.217 \mathrm{E}-08$ & $-3.045 \mathrm{E}-10$ & $-4.793 \mathrm{E}-08$ \\
$-4.503 \mathrm{E}-08$ & $-8.661 \mathrm{E}-10$ & $2.319 \mathrm{E}-08$ & $-6.483 \mathrm{E}-08$ \\
$-3.089 \mathrm{E}-08$ & $7.593 \mathrm{E}-08$ & $-1.784 \mathrm{E}-09$ & $-3.957 \mathrm{E}-08$ \\
$-2.774 \mathrm{E}-08$ & $4.721 \mathrm{E}-08$ & $2.202 \mathrm{E}-08$ & $1.032 \mathrm{E}-08$ \\
$-2.551 \mathrm{E}-08$ & $3.161 \mathrm{E}-08$ & $-2.341 \mathrm{E}-08$ & $-4.828 \mathrm{E}-08$ \\
$-9.630 \mathrm{E}-09$ & $6.075 \mathrm{E}-08$ & $3.480 \mathrm{E}-08$ & $1.243 \mathrm{E}-07$ \\
$-1.188 \mathrm{E}-08$ & $1.022 \mathrm{E}-08$ & $6.543 \mathrm{E}-08$ & $3.216 \mathrm{E}-08$ \\
$6.240 \mathrm{E}-08$ & $-9.888 \mathrm{E}-10$ & $5.393 \mathrm{E}-08$ & $2.248 \mathrm{E}-08$ \\
\hline
\end{tabular}

Unit: $\mathrm{m}$ 


\subsubsection{Uncertainty that resulted from the calibration results of the laser} interferometer $\left(u_{5}\right)$

The HP frequency-stabilized laser was calibrated in our national measurement laboratory (Calibration NO. B960384). Its variable range of laser frequency is $2.8 \mathrm{MHz}$. The horizontal wavelength equals $0.6 \mu \mathrm{m}$ and the vertical wavelength is $0.6 \mu \mathrm{m}$ in a vacuum after calibration. The rectangular probability distribution was adopted in this article. It was assumed that the relative uncertainty of uncertainty is $20 \%$, the degree of freedom is $12.5\left(v_{5}\right)$.

$$
\begin{aligned}
& \left|\Delta \lambda_{0}\right|=\frac{c}{f^{2}}(\Delta f)=\frac{299792458}{(473612226.8)^{2}} \times 2.8=3.74 \times 10^{-9}(\mu \mathrm{m}) \\
& \text { Error } \delta_{1}=L \times \frac{\left|\Delta \lambda_{0}\right|}{\lambda_{0}}=88 \times \frac{3.74 \times 10^{-9}}{0.6329913821}=5.20 \times 10^{-7}(\mathrm{~mm})
\end{aligned}
$$

Here, $c$ is velocity of light, $F$ is laser frequency, $\Delta f$ is variable range of laser frequency, $\lambda_{0}$ is laser wavelength, $\Delta \lambda_{0}$ is variable range of laser wavelength, and $L$ is total length of measurement displacement.

$$
u_{5}=\frac{5.20 \times 10^{-7} / 2}{\sqrt{3}}=1.50 \times 10^{-7}(\mathrm{~mm})
$$

\subsubsection{Uncertainty that resulted from the air refraction changes due to temperature deviation $\left(u_{6}\right)$}

Owing to the effects of air, temperature and wave motion, the refractive index of air would be changed. The values of temperature, atmospheric pressure, and relative humidity in the laboratory are substituted into the modified formula, and then the modified wavelength can be obtained.

$$
\left\{\begin{array}{l}
C=\frac{10^{6}}{N+10^{6}} \\
N=0.3836391 P \times\left[\frac{1+10^{-6} \times P \times(0.817-0.0133 T)}{1+0.0036610 T}\right]-3.033 \times 10^{-3} \times H \times e^{0.057627 T}
\end{array}\right.
$$

Here, $C$ is the complementary factor, $T$ is temperature $\left({ }^{\circ} \mathrm{C}\right), P$ is atmospheric pressure (mmHg), and $H$ is humidity (\%).

If the room temperature is $23 \pm 1.5^{\circ} \mathrm{C}$, the atmospheric pressure is $750 \pm 20 \mathrm{mmHg}$ and relative humidity is $45 \pm 10 \%$, the complementary factor can be calculated as follows. The maximum complementary factor $C_{\max }$ is 0.99974365 and the minimum complementary factor $C_{\min }$ is 0.99972648 . Therefore, the maximum error of modified wavelength was

$$
\begin{gathered}
\lambda_{0} \times\left(C_{\max }-C_{\min }\right)=1.087 \times 10^{-5}(\mu \mathrm{m}) . \\
\text { Error } \delta_{2}=L \times \frac{\lambda_{0} \times\left(C_{\max }-C_{\min }\right)}{\lambda_{0}}=88 \times \frac{1.087 \times 10^{-5}}{0.6329913821}=1.51 \times 10^{-3}(\mathrm{~mm})
\end{gathered}
$$


It was assumed that relative uncertainty of uncertainty is $20 \%$, the degree of freedom is $12.5\left(v_{6}\right)$.

$$
u_{6}=\frac{1.51 \times 10^{-3} / 2}{\sqrt{3}}=4.36 \times 10^{-4}(\mathrm{~mm})
$$

\subsubsection{Uncertainty that resulted from Abbe's and Chosine's error $\left(u_{7}\right)$}

The Abbe's error was caused by rotation of the instrument's driver axis. The Chosine's error was induced by unparallel lines of the measured axis of laser and the vertical axis. The diameter of the laser beam on the reference mirror is $3 \mathrm{~mm}$. In the measurement displacement of 0-88 mm, the laser beam shift can be estimated from the displacement shift on the reference mirror. The value of the shift is smaller than \pm 0.125 times the diameter and the maximum shifted angle is $0.244\left(\tan ^{-1}(3 / 8 / 88)\right)$ degrees. Hence, the error of $\delta_{3}$ was defined as

$$
\begin{aligned}
& \text { Error } \delta_{3}=88 \times\left(1-\cos 0.244^{\circ}\right)=7.80 \times 10^{-4}(\mathrm{~mm}) \\
& u_{7}=\frac{7.80 \times 10^{-4}}{\sqrt{3}}=4.50 \times 10^{-4}(\mathrm{~mm}) .
\end{aligned}
$$

It was assumed that the relative uncertainty of uncertainty is $20 \%$, and the degree of freedom is $12.5\left(v_{7}\right)$.

\subsubsection{Uncertainty that resulted from the unparallel lines of the path of laser light} and the vertical axis $\left(u_{8}\right)$

In the range of 0-88 mm, unparallel errors between the path of the laser light and the vertical axis were estimated from the displacement shift on the reference mirror. The value of the shift is smaller than \pm 0.125 times the diameter, and the maximum shifted angle is 0.244 degrees. Hence, the error of $\delta_{4}$ was the same as that of $\delta_{3}$. It was assumed that the relative uncertainty of uncertainty is $20 \%$, and the degree of freedom is $12.5\left(v_{8}\right)$.

$$
u_{8}=\frac{7.80 \times 10^{-4}}{\sqrt{3}}=4.50 \times 10^{-4}(\mathrm{~mm})
$$

\subsubsection{Uncertainty that resulted from the repeatability of the measurement system $\left(u_{9}\right)$}

During the calibration, the readout of the laser was defined by the displacement of the Nano UTM. Therefore, the repeatability of the measurement system was calculated from thirty displacement measurements as shown in Table 3.

$$
u_{9}=\sqrt{\frac{\sum_{i=1}^{30} s_{i}^{2}}{30}}=0.00266(\mathrm{~mm})
$$


Table 3

Repeated data of measurement displacement.

\begin{tabular}{|c|c|c|c|c|c|c|c|c|c|}
\hline No. & Run 1 & Run 2 & Run 3 & $\begin{array}{l}\text { Standard } \\
\text { deviation }\end{array}$ & No. & Run 1 & Run 2 & Run 3 & $\begin{array}{l}\text { Standard } \\
\text { deviation }\end{array}$ \\
\hline 1 & -0.00012 & 0.00012 & -0.00002 & 0.00012 & 16 & -29.9986 & -30.0034 & -30.0033 & 0.00274 \\
\hline 2 & -1.99535 & -1.99979 & -1.99988 & 0.00259 & 17 & -31.9988 & -32.0033 & -32.0032 & 0.00257 \\
\hline 3 & -3.99498 & -3.99955 & -3.99966 & 0.00267 & 18 & -33.9982 & -34.0028 & -34.0029 & 0.00269 \\
\hline 4 & -5.99485 & -5.99947 & -5.99957 & 0.00270 & 19 & -35.9978 & -36.0026 & -36.0024 & 0.00272 \\
\hline 5 & -7.99503 & -7.99956 & -7.99964 & 0.00264 & 20 & -37.9984 & -38.0033 & -38.0033 & 0.00283 \\
\hline 6 & -9.99485 & -9.99958 & -9.99958 & 0.00273 & 21 & -39.9988 & -40.0037 & -40.0037 & 0.00283 \\
\hline 7 & -11.9951 & -11.9998 & -11.9997 & 0.00267 & 22 & -41.9996 & -42.0044 & -42.0044 & 0.00277 \\
\hline 8 & -13.9954 & -14.0001 & -14.0001 & 0.00271 & 23 & -44.0007 & -44.0055 & -44.0054 & 0.00274 \\
\hline 9 & -15.9965 & -16.0001 & -16.0001 & 0.00208 & 24 & -46.0018 & -46.0067 & -46.0067 & 0.00283 \\
\hline 10 & -17.9973 & -18.0018 & -18.0019 & 0.00263 & 25 & -48.0025 & -48.0073 & -48.0073 & 0.00277 \\
\hline 11 & -19.9981 & -20.0027 & -20.0026 & 0.00263 & 26 & -50.0023 & -50.0072 & -50.0072 & 0.00283 \\
\hline 12 & -21.9985 & -22.0032 & -22.0031 & 0.00269 & 27 & -52.0023 & -52.0072 & -52.0073 & 0.00286 \\
\hline 13 & -23.9992 & -24.0037 & -24.0039 & 0.00266 & 28 & -54.0027 & -54.0077 & -54.0076 & 0.00286 \\
\hline 14 & -25.9995 & -26.0041 & -26.0041 & 0.00266 & 29 & -56.0022 & -56.0072 & -56.0071 & 0.00286 \\
\hline 15 & -27.9989 & -28.0036 & -28.0035 & 0.00269 & 30 & -58.0021 & -58.0071 & -58.0069 & 0.00283 \\
\hline
\end{tabular}

Unit: mm

The uncertainty that resulted from the repeatability of the measurement system $\left(u_{9}\right)$ is 2.66 $\times 10^{-3} \mathrm{~mm}$, and the degree of freedom is $90\left(v_{9}\right)$.

\subsubsection{Uncertainty that resulted from the deviation of the measurement data} without correction $\left(u_{10}\right)$

The indication error of measurement displacement was not modified in this system. The maximum indication error was $0.01989 \mathrm{~mm}$ in the calibrated range. It was assumed that the relative uncertainty of uncertainty is $20 \%$, and the degree of freedom is $12.5\left(v_{10}\right)$.

$$
u_{10}=\frac{1.989 \times 10^{-2} / 2}{\sqrt{3}}=5.74 \times 10^{-3}(\mathrm{~mm})=5.74 \times 10^{-6}(\mathrm{~m})
$$

\subsubsection{Combined standard uncertainty $\left(u_{L}\right)$ and expanded uncertainty $\left(U_{L}\right)$}

It was assumed that these sources of uncertainties are linear-independent. The individual terms are collected and substituted into the expression to obtain the combined standard uncertainty $\left(u_{L}\right)$.

$$
\begin{gathered}
u_{L}=\sqrt{u_{4}^{2}+u_{5}^{2}+u_{6}^{2}+u_{7}^{2}+u_{8}^{2}+u_{9}^{2}+u_{10}^{2}}=6.42 \times 10^{-6}(\mathrm{~m}) \\
\text { Effective degrees of freedom } v_{L}=\frac{u_{c}{ }^{4}}{\sum_{i=4}^{10} \frac{\left(C_{i} \times u_{i}\right)^{4}}{v_{i}}}=18.8
\end{gathered}
$$


When the effective degree of freedom is 18.8 , the coverage factor $k$ is equal to 2.10 with a $95 \%$ confidence level from the t-distribution. ${ }^{(2)}$ The expanded uncertainty $\left(U_{L}\right)$ can be obtained from the coverage factor and combined standard uncertainty.

$$
U_{L}=k \times u_{L}=2.1 \times 6.42 \times 10^{-6}(\mathrm{~m})=1.4 \times 10^{-2}(\mathrm{~mm})
$$

\subsection{Uncertainty evaluation of Young's modulus and mechanical properties of PDMS}

It has been pointed out earlier in this paper that the combined standard uncertainty of force and displacement measurement can be calculated from reasonable factors. Based on the above results, the uncertainty of loading and displacement can be used to estimate the uncertainty of Young's modulus. The Young's modulus of pure PDMS was measured in this study.

\subsubsection{Uncertainty evaluation of Young's modulus}

The formula of Young's modulus is

$$
E=\frac{\sigma}{\varepsilon}=\frac{\frac{F}{A}}{\frac{\Delta L}{L}},
$$

here $\sigma$ is the stress $\left(\mathrm{mN} / \mathrm{mm}^{2}\right), \varepsilon$ is the strain, $F$ is loading force $(\mathrm{mN}), A$ is the area of cross section $\left(\mathrm{mm}^{2}\right), L$ is the gauge length $(\mathrm{mm})$, and the $\Delta L$ is the extension length of gauge length $(\mathrm{mm})$.

Therefore, the uncertainty of Young's modulus can be affected by these four items including (i) the uncertainty of loading force, (ii) the uncertainty that resulted from the area of cross section, (iii) the uncertainty that resulted from the gauge length, and (iv) the elongation of gauge length.

(i) Uncertainty of loading force $\left(u_{F}\right)$

In the tensile test, the standard uncertainty of loading force $\left(u_{F}\right)$ is $0.207 \mathrm{mN}$ and the effective degree of freedom is $107225.5\left(v_{F}\right)$ from Table 1.

(ii) Uncertainty that resulted from the area of cross section $\left(u_{A}\right)$

The micrometer (Mitutoyo/MDC-1" PJ/293-340-70) was used to measure the thickness of the specimens. From the report, the expanded uncertainty of micrometer was $1.27 \times 10^{-3} \mathrm{~mm}$ and the standard uncertainty $\left(u_{m}\right)$ was $6.35 \times 10^{-4} \mathrm{~mm}$. The digital caliper (Mitutoyo/CD-8" CS/0041756) was used to measure the width of the specimens and its standard uncertainty $\left(u_{d}\right)$ was $0.01 \mathrm{~mm}$. The average thickness $(\bar{t})$ of the specimen is $0.310 \mathrm{~mm}$ and the standard deviation $\left(S_{t}\right)$ is $0.0036 \mathrm{~mm}$. The average width $(\bar{w})$ of the specimen is $2.08 \mathrm{~mm}$ and the standard deviation $\left(S_{w}\right)$ is $0.04 \mathrm{~mm}$. Therefore, the uncertainty that resulted from the area of cross section can be calculated as

$$
u_{A}=\sqrt{\left(u_{w} \times \bar{t}\right)^{2}+\left(u_{t} \times \bar{w}\right)^{2}}=1.49 \times 10^{-2}\left(\mathrm{~mm}^{2}\right),
$$

here $u_{w}=\sqrt{\left(S_{w}\right)^{2}+\left(u_{d}\right)^{2}}, u_{t}=\sqrt{\left(S_{t}\right)^{2}+\left(u_{m}\right)^{2}}$, and the degree of freedom is $9.86\left(v_{A}\right)$. 
(iii) Uncertainty that resulted from the gauge length $\left(u_{L}\right)$

The digital caliper was also used to measure the gauge length of the specimens. From the report (H96-06-153-01), its expanded uncertainty was $0.02 \mathrm{~mm}$, so the standard uncertainty of the digital caliper was $0.01 \mathrm{~mm}$. The gauge lengths of the specimens were measured three times and the values were 18.95, 19.02 and $19.04 \mathrm{~mm}$. The average gauge length was approximately $19.00 \mathrm{~mm}$ and the standard deviation $\left(S^{*}\right)$ was 0.047 . The uncertainty of the gauge length $\left(u_{L}\right)$ can be calculated using eq. (20), and then the effective degree of freedom is $2.57\left(v_{L}\right)$.

$$
u_{L}=\sqrt{u_{\mathrm{d}}^{2}+\left(\frac{S^{*}}{\sqrt{3}}\right)^{2}}=2.89 \times 10^{-2}(\mathrm{~mm})
$$

(iv) Elongation of gauge length $\left(u_{\Delta L}\right)$

When a $200-\mathrm{mN}$ load is applied to the specimen, the elongation is $4.325 \mathrm{~mm}$. From eq. (16), the standard uncertainty of the displacement measurement $\left(u_{L}\right)$ is $6.42 \times 10^{-3} \mathrm{~mm}$, and the degree of freedom is $18.8\left(v_{\Delta L}\right)$.

\subsubsection{Combined standard uncertainty $\left(u_{E}\right)$ and expanded uncertainty $\left(U_{E}\right)$}

The combined uncertainty $\left(u_{\mathrm{E}}\right)$ of Young's modulus was calculated as

$$
\begin{aligned}
u_{E} & =\sqrt{\sum_{i=1}^{4}\left(\frac{\partial E}{\partial c_{i}}\right) u^{2}\left(x_{i}\right)} \\
& =\sqrt{\left(C_{F}+u_{F}\right)^{2}+\left(C_{A}+u_{A}\right)^{2}+\left(C_{L}+u_{L}\right)^{2}+\left(C_{\Delta L}+u_{\Delta L}\right)^{2}}=31.63\left(\mathrm{mN} / \mathrm{mm}^{2}\right)
\end{aligned}
$$

here the sensitivity coefficients of uncertainties are

$$
\begin{aligned}
& C_{F}=\frac{\partial E}{\partial F}=\frac{L}{A \times \Delta L}=6.81, C_{A}=\frac{\partial E}{\partial A}=\frac{F \times L}{A^{2} \times \Delta L}=2113.23 \\
& C_{L}=\frac{\partial E}{\partial L}=\frac{F}{A \times \Delta L}=71.72, C_{\Delta L}=\frac{\partial E}{\partial \Delta L}=\frac{F \times L}{A \times \Delta L^{2}}=315.06 .
\end{aligned}
$$

The effective degree of freedom is

$$
v_{\text {eff }}=\frac{u_{E}{ }^{4}}{\frac{\left(C_{F} \times u_{F}\right)^{4}}{v_{F}}+\frac{\left(C_{A} \times u_{A}\right)^{4}}{v_{A}}+\frac{\left(C_{L} \times u_{L}\right)^{4}}{v_{L}}+\frac{\left(C_{\Delta L} \times u_{\Delta L}\right)^{4}}{v_{\Delta L}}}=10.04 \approx 10 .
$$

It is observed that the uncertainty of the cross-sectional area is one of the most significant factors for the Young's modulus. When the effective degree of freedom is 10, the coverage factor $k$ is equal to 2.23 with a $95 \%$ confidence level from the t-distribution. ${ }^{(2)}$ The expanded uncertainty $\left(U_{\mathrm{E}}\right)$ can be obtained from the coverage factor and combined standard uncertainty.

$$
U_{\mathrm{E}}=2.23 \times 31.63=70.53\left(\mathrm{mN} / \mathrm{mm}^{2}\right)
$$


The Young's modulus of the specimen was defined as

$$
E=\frac{F \times L}{A \times \Delta L}=\frac{200 \times 19}{0.6448 \times 4.325}=1362.61\left(\mathrm{mN} / \mathrm{mm}^{2}\right)
$$

The relative expanded uncertainty $U_{\mathrm{E}} / E$ is $5.18 \times 10^{-2}$.

Figure 5 shows the stress and strain curves of PDMS at a curing temperature of $100^{\circ} \mathrm{C}$. The experiments were tested on at least three specimens. Since PDMS has the non-linear elastic behavior, the Young's modulus was determined at the range of small strain (within the strain of 0.05). The average Young's modulus of the three specimens is $1.37 \mathrm{MPa}$ as shown in Fig. 6. For one of the specimens, the relative expanded uncertainty is $5.18 \%$ when the Young's modulus of PDMS is $1.36 \mathrm{MPa}$. The loading and unloading curves of PDMS are shown in Fig. 7. It is observed that the energy dissipation of PDMS is small.

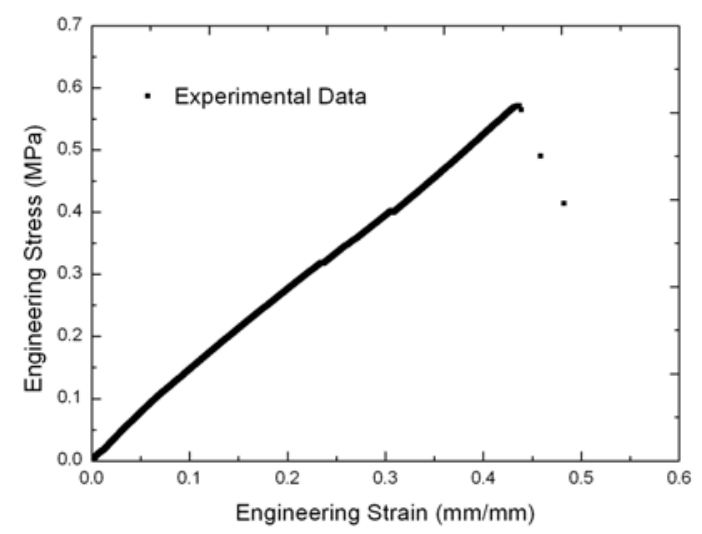

Fig. 5. Stress-strain curve of PDMS material.

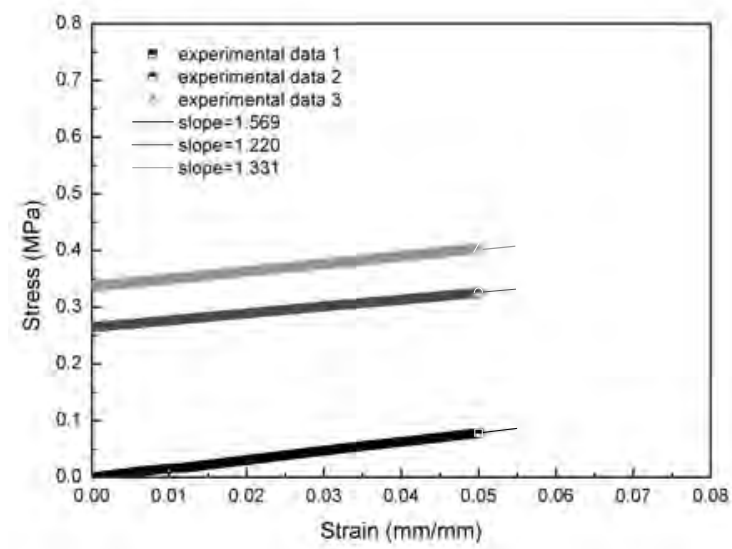

Fig. 6. Young's modulus of three specimens. 


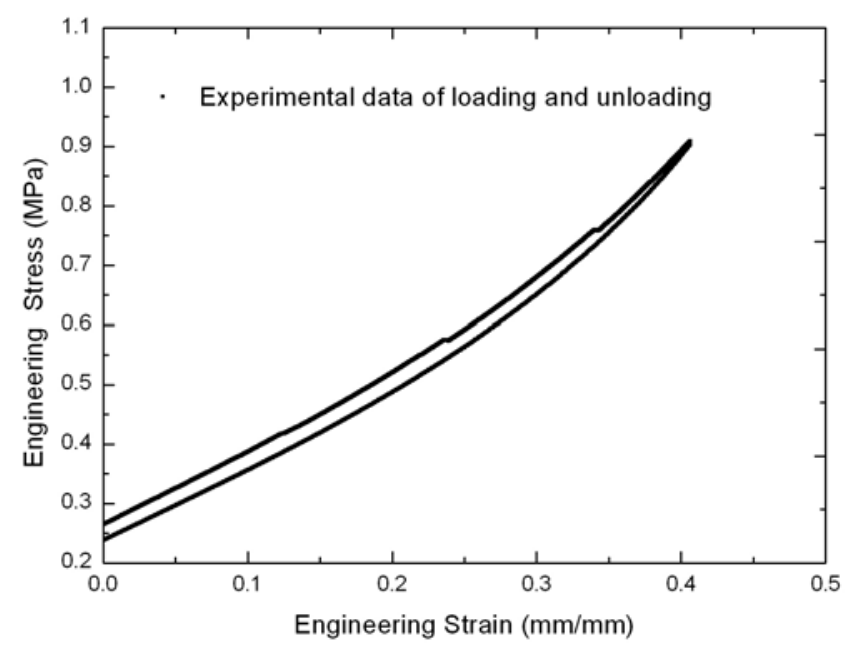

Fig. 7. Loading and unloading curves of PDMS.

\section{Conclusion}

In conclusion, the uncertainties of force and displacement measurements for the Nano Universal Testing System were investigated. Traceable standard weights and optical interferometer were used to calibrate the force and displacement measurements, respectively. The result shows that the relative expanded uncertainty of the force corresponding to a level of confidence of $95 \%$ within the range of 10 to $200 \mathrm{mN}$ is 2.07 $\times 10^{-3} \mathrm{mN}$. The expanded uncertainty of displacement measurement within the range of 0 to $88 \mathrm{~mm}$ is $1.4 \times 10^{-5} \mathrm{~m}$ with a $95 \%$ confidence level. It is reasonable to calculate the uncertainty of Young's modulus from the expanded uncertainties of force and displacement measurements. The uncertainty of the cross-sectional area is the major factor for the deviation of Young's modulus.

\section{References}

1 Customer Care Kit, Nano Bionix Universal Testing System, MTS Nano Instrument Innovation Center.

2 ISO, Guide to the Expression of Uncertainty in Measurement (corrected and reprinted, 1995).

3 B. H. Jo, L. M. Van Lerberghe, K. M. Motsegood and D. J. Beebe: J. Microelectromech. Sys. 9 (2000) 76.

4 T. Fujii: Microelectron. Eng. 61-62 (2002) 907.

5 I. Park, J. Cheng, A. P. Pisano, E. S. Lee and J. H. Jeong: Appl. Phys. Lett. 90 (2007) 093902. 